\title{
Prognostic role of systemic immune-inflammation index in solid tumors: a systematic review and meta-analysis
}

\author{
Jie-Hui Zhong', Dan-Hui Huang ${ }^{1}$ and Zi-Yu Chen ${ }^{1}$ \\ ${ }^{1}$ Department of Clinical Medicine, The First Clinical Medical College, Southern Medical University, Guangzhou, 510515, China \\ Correspondence to: Jie-Hui Zhong, email: zhongjiehui@126.com
}

Keywords: systemic immune-inflammation index, solid tumors, meta-analysis

Received: April 05, 2017

Accepted: June 10, 2017

Published: June 29, 2017

Copyright: Zhong et al. This is an open-access article distributed under the terms of the Creative Commons Attribution License 3.0 (CC BY 3.0), which permits unrestricted use, distribution, and reproduction in any medium, provided the original author and source are credited.

\section{ABSTRACT}

Background: Inflammation may play an important role in cancer progression, and a higher systemic immune-inflammation index (SII) has been reported to be a poor prognostic marker in several malignancies. However, the results of published studies are inconsistent.

Materials and Methods: A systematic review of databases was conducted to search for publications regarding the association between blood SII and clinical outcome in solid tumors with a date up to February 12, 2017. The primary outcome was overall survival (OS) and the secondary outcomes were progression-free survival (PFS) and cancer-specific survival (CSS). Pooled hazard ratios (HRs) and $\mathbf{9 5 \%}$ confidence intervals (CIs) were used to assess the strength of the association between blood SII and clinical outcome in solid tumors.

Results: A total of $\mathbf{1 5}$ articles were included in the analysis. Overall, systemic immune-inflammation index greater than the cutoff predicted poor overall survival $(H R=1.55,95 \% C I=1.27-1.88 ; P<0.001)$. Subgroup analyses revealed that high systemic immune-inflammation index indicated a worse overall survival in hepatocellular carcinoma $(P<0.001)$, urinary cancers $(P<0.001)$, gastrointestinal tract cancers $(P=0.02)$, small cell lung cancer $(P<0.05)$ and acral melanoma $(P<0.001)$. Hazard ratio for systemic immune-inflammation index greater than the cutoff for cancer-specific survival was $1.44(P<0.05)$.

Conclusions: Elevated systemic immune-inflammation index is associated with a worse overall survival in many solid tumors. The systemic-inflammation index can act as a powerful prognostic indicator of poor outcome in patients with solid tumors.

\section{INTRODUCTION}

Inflammatory reaction play crucial role in shaping tumor development in many aspects, ranging from tumor initiation to tumor metastasis [1]. Inflammatory related peripheral cells (neutrophils, lymphocytes and platelets) derived from the peripheral blood were significantly associated with tumor progression in various tumors [2-4]. Moreover, inflammatory indexes (II) obtained with different combinations of these factors, such as neutrophil to lymphocyte ratio (NLR) and platelet to lymphocyte ratio (PLR), have been investigated as useful prognostic factors in various malignant solid tumors [5-7]. A novel inflammatory index, the systemic immune inflammation index (SII), defined as follows: $\mathrm{SII}=\mathrm{P} * \mathrm{~N} / \mathrm{L}$, where $\mathrm{P}$, $\mathrm{N}$ and $\mathrm{L}$ were the peripheral platelet, neutrophil and lymphocyte counts [8], was recently investigated as a prognostic marker in various malignancies. Geng et al. [9] suggested that SII was superior to the other systemic inflammation index such as PLR and NLR, and served as a more objective marker that reflects the balance between host inflammatory and immune response status.

The goal of the present study was to conduct a metaanalysis to investigate the association between peripheral blood SII in solid tumors and clinical outcome. Our hypothesis was that elevated SII correlates with worse OS and may thus serve as a readily available and inexpensive prognostic marker in clinical practice. 


\section{MATERIALS AND METHODS}

\section{Study identification and selection}

Studies regarding the association between SII and clinical outcome in solid tumors published before February 12, 2017 were included through PubMed, Embase and Cochrane library searching by using the following terms and key words: (systemic immune inflammation index OR SII) AND cancer. The criteria used for the study selection were as followed: 1) studies were concerned about the prognostic impact of SII; 2) A hazard ratio (HR) and 95\% confidence interval (CI) for overall survival (OS), cancer-specific survival (CSS) and progression-free survival (PFS) was available; 3 ) there were no overlapping data. Two of the authors (Jiehui Zhong, Danhui Huang) evaluated the eligibility of all studies independently collected from the databases based on the selection criteria. The Newcastle-Ottawa Scale (NOS) was used to assess study quality, which consists of three parameters of quality: selection ( $0-4$ points), comparability (0-2 points), and outcome assessment (0-3 points) (Supplementary Table 1$)$. Studies with the scores $\geq 6$ were assigned as high-quality studies.

\section{Data extraction}

Information was carefully extracted from all the eligible studies independently by three investigators according to the selection criteria listed above. The following data were collected: first author's name, publication year, country, type of publication (abstract, full text), number of patients included in analysis, disease site, disease stage (metastatic, nonmetastatic, mixed [metastatic and nonmetastatic]), collection of data (prospective, retrospective), cutoff defining high SII used for peripheral blood SII, and hazard ratios and $95 \%$ confidence interval (CI) for OS, CSS, or RFS as applicable. Hazard ratios based on multivariable analyses other than univariate analyses were extracted prior. We did not require a minimum number of patients to be included in our meta-analysis.

\section{Statistical analysis}

Meta-analysis was conducted using RevMan 5.3 analysis software (Cochrane Collaboration, Copenhagen, Denmark). Estimation of hazard ratios was pooled using the random-effect model or the fixed-effect model. Heterogeneity assumption was tested by a chi-squarebased $Q$ test. Proportion of the total variation across studies due to heterogeneity was checked by $I$-square statistics [10]. A $P$-value of $>0.05$ for the $Q$-test indicated a lack of heterogeneity among studies, so that the pooled HR estimate of each study was calculated by the fixed-effect model [11]. Otherwise, the random-effect model was used [12]. Subgroup analyses were conducted according to disease site (i.e., hepatocellular carcinoma or urinary cancers), disease stage (metastatic/mixed or nonmetastatic), cutoffs for SII (SII $\geq 572$ or SII $<572$ ), data collection (prospective or retrospective), analysis of HR (multivariable or univariable) and article type (abstract or full paper). Differences between the subgroups were assessed using methods described by Deeks et al. [13]. The effect of SII cutoff on the hazard ratio for OS was evaluated by meta-regression analysis. Publication bias is the tendency on the parts of investigators, reviewers, and editors to submit or accept manuscripts for publication based on the direction or strength of the study findings [14]. An estimate of potential publication bias was carried out by the funnel plot, in which the standard error of $\log$ (HR) of each study was plotted against its $\log$ (HR). Funnel plot asymmetry was further assessed by the method of Egger's linear regression test $(P<0.05$ was considered a significant publication bias) [15].

\section{RESULTS}

\section{Extraction process and study characteristics}

The selection procedure was listed in Figure 1. Totally, 837 articles of interest were found after preliminary search, and 40 of them have relevant content on the association between SII and clinical outcome in solid tumors. Among them, 25 studies met the exclusion criterion due to duplication or lack of available data. Hence, 15 publications $[8,9,16-28]$ including 16 studies (4875 patients) were selected. The characteristics of the studies included were shown in Table 1. Of them, there were 5 hepatocellular carcinoma studies, 2 esophageal squamous cell carcinoma studies, 2 gastric cancer studies, 1 renal cell cancer study, 1 malignant obstructive jaundice study, 1 small cell lung cancer studies, 1 colorectal cancer study, 1 biliary tract cancer study, and 1 metastatic castration-resistant prostate cancer study and 1 acral melanoma study. The quality of all 16 studies ranged from 5 to 7 (Supplementary Table 1), indicating that most studies included were high quality.

\section{Overall survival}

15 studies including 4577 patients reported HR for OS. The median cutoff for high SII was 572 (range $=300-1600$ ). SII greater than the cutoff predicted poor OS $(\mathrm{HR}=1.55,95 \% \mathrm{CI}=1.27-1.88 ; P<0.001$, Figure 2). However, this result is with significant betweenstudy heterogeneity (I-square $=63 \%, P<0.001$ ). The influence of SII on OS among cancer subgroups is shown in Table 2. High SII was associated with significantly worse OS for acral melanoma, hepatocellular carcinoma, urinary cancers, small cell lung cancer, gastrointestinal tract cancers $(\mathrm{HRs}=2.54,2.08,1.82,1.38,1.21$, respectively). The influence of SII on OS among different cancer stages is shown in Table 2. The HRs were $1.65(95 \% \mathrm{CI}=1.28-2.13)$ 
Table 1: Characteristics of studies included in the meta-analysis

\begin{tabular}{|c|c|c|c|c|c|c|c|c|c|}
\hline Author & Study Period & $\begin{array}{c}\text { Data } \\
\text { collection }\end{array}$ & Ethnicity & Stage & $\begin{array}{c}\text { Disease } \\
\text { site }\end{array}$ & Number & Cut-off & $\begin{array}{l}\text { Reported } \\
\text { endpoints }\end{array}$ & $\begin{array}{c}\text { Follow-up } \\
\text { Median } \\
\text { (month) }\end{array}$ \\
\hline Hu 20141 & $2005-2006$ & retrospective & China & mixed & $\mathrm{HCC}$ & 133 & 330 & OS, & 61.3 \\
\hline Hu 20142 & 2010-2011 & retrospective & China & mixed & $\mathrm{HCC}$ & 123 & 330 & OS & 28.8 \\
\hline Lolli 20161 & 2006-2014 & retrospective & Italy & metastatic & $\mathrm{RCC}$ & 335 & 730 & OS, PFS & 49 \\
\hline Lolli 20162 & 2011-2015 & retrospective & Italy & metastatic & $\mathrm{mCRPC}$ & 230 & 535 & OS & 29 \\
\hline Yang 2015 & 2009-2015 & retrospective & China & mixed & $\mathrm{HCC}$ & 189 & 300 & OS & 19.8 \\
\hline Passardic 2016 & $2007-2012$ & prospective & Italy & metastatic & $\mathrm{CRC}$ & 289 & 730 & OS, PFS & 36 \\
\hline Feng 2016 & 2005-2008 & retrospective & China & nonmetastatic & ESCC & 298 & 410 & CSS & NR \\
\hline На 2016 & 2004-2009 & retrospective & Korea & NR & BTC & 158 & 572.38 & OS & 95.3 \\
\hline Geng 2016 & 2002-2012 & retrospective & China & nonmetastatic & ESCC & 916 & 307 & OS & 39 \\
\hline Jin 2016 & $2012-2016$ & retrospective & China & NR & MOJ & 33 & 644 & OS & 10 \\
\hline Hong 2015 & $2000-2012$ & retrospective & China & mixed & SCLC & 919 & 1600 & OS & NR \\
\hline Liu 2015 & 2005-2010 & retrospective & China & nonmetastatic & $\mathrm{GC}$ & 455 & 660 & OS & 25 \\
\hline Gardini 2016 & $2012-2015$ & retrospective & Italy & mixed & $\mathrm{HCC}$ & 56 & 360 & OS, PFS & NR \\
\hline Huang 2016 & 2013-2014 & retrospective & China & nonmetastatic & GC & 445 & 572 & OS & 54.6 \\
\hline Gao 2016 & 2014-2015 & retrospective & China & mixed & $\mathrm{HCC}$ & 183 & 330 & OS & NR \\
\hline Yu 2016 & NR & NR & China & NR & $\mathrm{AM}$ & 113 & 615 & OS & NR \\
\hline
\end{tabular}

HCC, hepatocellular carcinoma; MCRPC, metastatic castration-resistant prostate cancer; RCC, renal cell cancer; ESCC, esophageal squamous cell carcinoma; BTC, biliary tract cancer; MOJ, malignant obstructive jaundice; SCLC, small cell lung cancer ; CRC, colorectal cancer; GC, gastric cancer; AM, acral melanoma; OS, overall survival; PFS, progression-free survival; CSS, cancer-specific survival; NR, not reported.

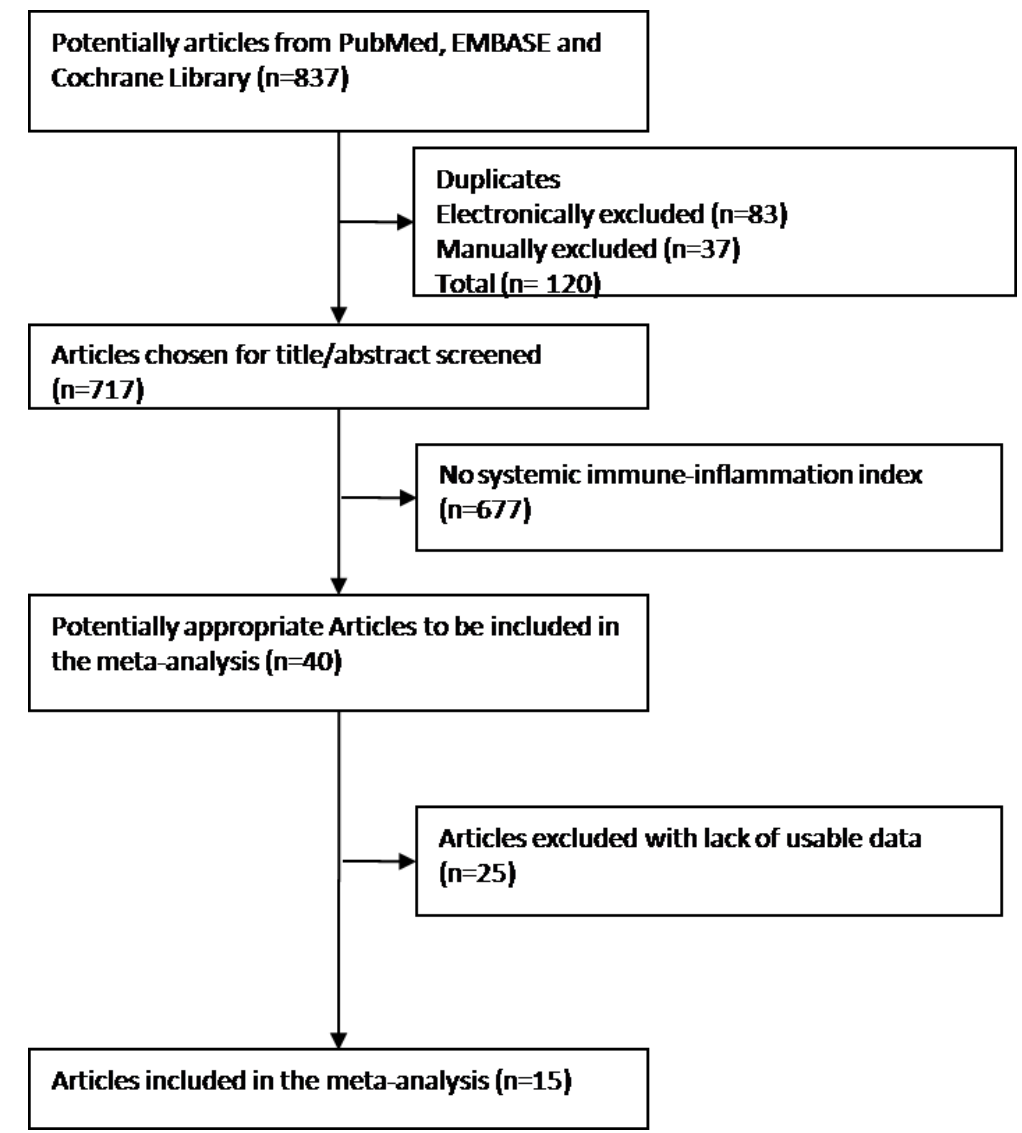

Figure 1: Flow diagram of included studies for this meta-analysis. 
Table 2: Subgroup analyses of overall survival

\begin{tabular}{|c|c|c|c|c|c|}
\hline Subgroup & $\begin{array}{c}\text { Number of } \\
\text { studies }\end{array}$ & HR (95\% CI) & $\boldsymbol{P}$ & $\boldsymbol{P}_{h}$ & $\begin{array}{l}P \text { for subgroup } \\
\text { difference }\end{array}$ \\
\hline Disease site & & & & & 0.001 \\
\hline Gastrointestinal tract cancers & 4 & $1.21(1.03-1.41)$ & 0.02 & 0.09 & \\
\hline Urinary cancers & 2 & $1.82(1.43-2.32)$ & $<0.001$ & 0.93 & \\
\hline Hepatocellular carcinoma & 5 & $2.08(1.52-2.85)$ & $<0.001$ & 0.15 & \\
\hline Small cell lung cancer & 1 & $1.38(1.02-1.85)$ & 0.03 & - & \\
\hline Acral melanoma & 1 & $2.54(1.50-4.31)$ & $<0.001$ & - & \\
\hline Other & 2 & $1.84(0.35-9.63)$ & 0.47 & 0.04 & \\
\hline Disease stage & & & & & 0.60 \\
\hline Metastatic/mixed & 9 & $1.65(1.28-2.13)$ & 0.0001 & 0.01 & \\
\hline Nonmetastatic & 4 & $1.48(1.06-2.05)$ & 0.02 & 0.02 & \\
\hline Cutoffs for SII & & & & & 0.31 \\
\hline$\geq 572$ & 8 & $1.42(1.08-1.87)$ & 0.01 & 0.002 & \\
\hline$<572$ & 7 & $1.76(1.29-2.41)$ & $<0.001$ & 0.02 & \\
\hline Analysis of HR & & & & & 0.10 \\
\hline Multivariable & 14 & $1.59(1.31-1.93)$ & $<0.001$ & $<0.001$ & \\
\hline Univariable & 1 & $0.75(0.32-1.78)$ & 0.52 & - & \\
\hline Data collection & & & & & 0.02 \\
\hline Prospective & 1 & $0.84(0.53-1.33)$ & 0.46 & - & \\
\hline Retrospective & 13 & $1.56(1.28-1.89)$ & $<0.001$ & 0.005 & \\
\hline Article type & & & & & 0.06 \\
\hline Abstract & 1 & $2.54(1.50-4.31)$ & $<0.001$ & - & \\
\hline Full paper & 14 & $1.49(1.23-1.81)$ & $<0.001$ & 0.002 & \\
\hline
\end{tabular}

The subgroup "gastrointestinal tract cancers" includes 1 esophageal squamous cell carcinoma studies, 1 colorectal cancer study and 2 gastric cancer studies; The subgroup "urinary cancers" includes 1 renal cell cancer study and 1 metastatic castration-resistant prostate cancer study; The subgroup "other" includes 1 malignant obstructive jaundice study and 1 biliary tract cancer study. $P_{h}, P$-values for heterogeneity from $Q$ test.

$\begin{array}{lrrrrrr}\text { Study or Subgroup } & \text { log[Hazard Ratio] } & \text { SE } & \text { Weight } & \begin{array}{c}\text { Hazard Ratio } \\ \text { IV, Random, 95\% Cl }\end{array} & \text { Hazard Ratio } \\ \text { IV, Random, 95\% Cl }\end{array}$

Figure 2: Forest plots of studies evaluating the association between SII and overall survival. The center of each square represents the HR, the area of the square is the number of sample and thus the weight used in the meta-analysis, and the horizontal line indicates the $95 \% \mathrm{CI}$. 
for metastatic/mixed diseases, $1.48(95 \% \mathrm{CI}=1.06-2.05)$ for nonmetastatic diseases. The subgroups analyses according to cutoffs for SII, data collection, analysis of HR, article type regarding the effect of SII on OS is shown in Table 2. Meta-regression scatter plot is shown in Figure 3. Overall, there was no association between SII cutoff and the HR for OS $(P=0.519)$. Sensitivity analyses investigating the influence by omitting one study at a time and calculating the combined HRs. Any single study did not substantially affect the pooled HRs when deleted from the whole study. Begg's funnel plot and Egger's test were conducted to estimate the publication bias of studies. There was no publication bias because of bias exploration funnel plots demonstrated symmetry (Figure 4). Egger's test also validates little publication bias $(P=0.123)$.

\section{Progression-free survival}

Three studies including 680 patients reported HRs for PFS. Overall, SII over the cutoff was not associated with a hazard ratio for worse PFS outcome. The HR for PFS was $1.31(95 \% \mathrm{CI}=0.76-2.27, P=0.33$, Table 3$)$.

\section{Cancer-specific survival}

Only one study comprising 298 patients reported hazard ratios for CSS. Overall, SII over the cutoff was associated with a hazard ratio for worse CSS outcome. The HR for CSS was $1.44(95 \% \mathrm{CI}=1.04-1.99, P=0.03$, Table 3).

\section{DISCUSSION}

Many recent studies [8, 9, 16, 20-22, 24, 25, 27, 28] have suggested that an elevated SII is associated with poor overall survival or progression free survival of patients with cancers. While several studies [18, 19, 23, 26] show that SII is not significantly associated with overall survival. Thus the results of published studies are inconsistent. Here we undertook a meta-analysis of 16 studies including 4875 patients with solid tumors to assess whether SII was associated with prognosis of solid tumor. Overall, we found a significant association between elevated SII and poor survival. Consistent results were observed in various cancer subgroups, including hepatocellular carcinoma, gastrointestinal tract cancers, urinary cancers, small cell lung cancer and acral melanoma. Differences in HRs were observed between cancer sites and may be the result of inflammation playing different roles in different types of cancer. In addition, a trend for the association of high SII with worse OS was greater for metastatic/mixed cases than that in the nonmetastatic diseases. This may reflect either increased tumor burden or a long term inflammatory process [29]. The cutoff of SII in included studies didn't reach a standard point, and the method to determine the cutoffs is not described in many studies. Cutoffs of SII varied among the included studies, however, metaregression analysis showed that there was no association between SII cutoff and reported HR for OS. Thus, it was unlikely to influence our results. However, the cutoff value must be established in one cohort of patients and tested in

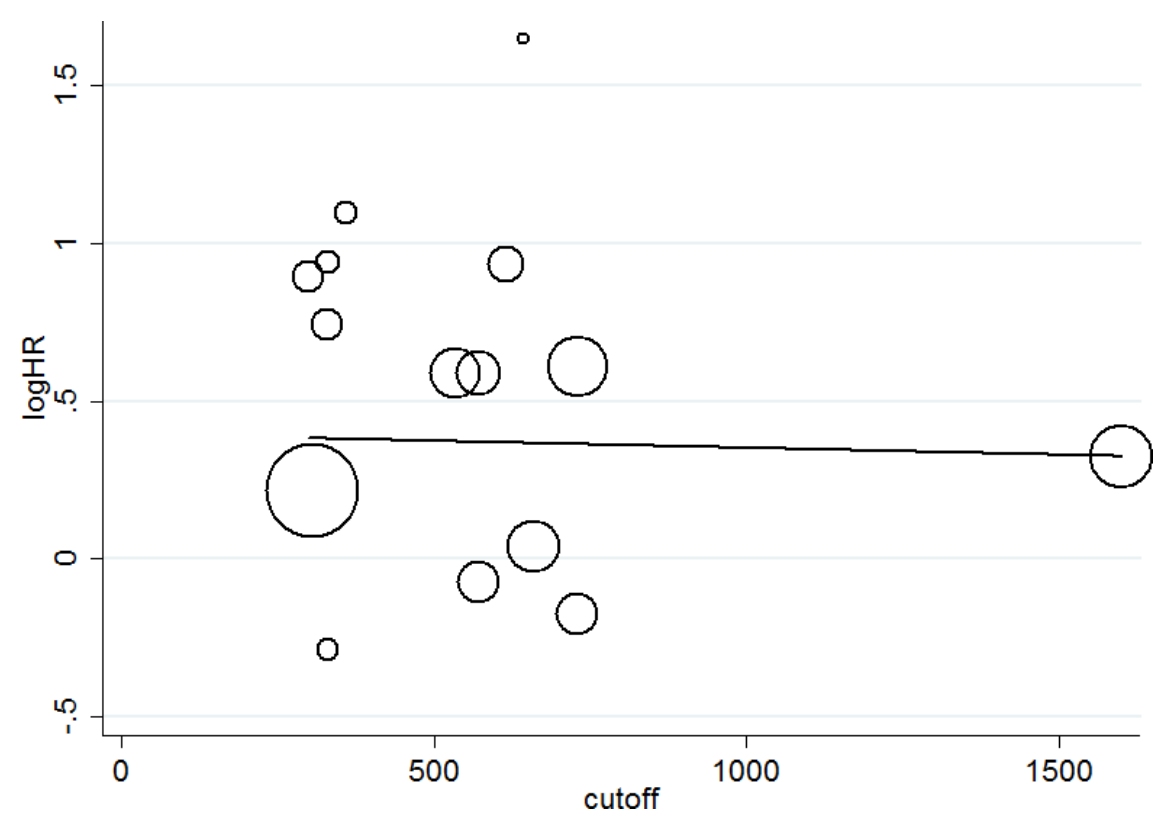

Figure 3: Univariate meta-regression exploring the association of the cutoff used to define SII and the hazard ratio for overall survival. 
Table 3: Subgroup analyses of secondary outcomes

\begin{tabular}{ccccc}
\hline Secondary outcomes & Num of studies & HR((95\% CI) & $\boldsymbol{P}$ & $\boldsymbol{P}_{h}$ \\
\hline PFS & 3 & $1.31(0.76-2.27)$ & 0.33 & 0.005 \\
CSS & 1 & $1.44(1.04-1.99)$ & 0.03 & - \\
\hline
\end{tabular}

PFS, progression-free survival; CSS, cancer-specific survival; $P \mathrm{~h}, P$-values for heterogeneity from $Q$ test.

another and the number of patients in each group needs to be considered in the statistical analysis [30].

Several studies may give explanations for the prognostic values of SII in tumors: Neutrophils activate endothelium and parenchymal cells via soluble factors secretion, enhancing circulating tumor cell adhesion in distant sites [31-33]. In addition, clinical studies have suggested that increased numbers of circulating neutrophils are associated with adverse prognosis in patients with cancers [34]. The effects of platelets on metastatic have been attributed to their ability to promote adhesion or to their capacity to prevent cell death. This is owing to the physical shield around tumor cells they formed [35]. Therefore, platelets may contribute to progression and metastasis of cancers. Lymphocytes play a crucial role in cancer immune surveillance and defense via cytotoxic cell death and inhibition tumor cell proliferation and migration [4]. Thus, due to high levels of neutrophils and platelets while low level of lymphocytes, a higher SII usually indicates a stronger inflammatory and a weaker immune response in patients. It may be associated with invasion and metastasis of tumor cells and hence lead to poor survival.
Some limitations of our meta-analysis existed. Firstly, the number of published studies included was not large enough for subgroup analysis. Secondly, only studies reporting HR and $95 \%$ confidence interval was included in our meta-analysis. Thus, further bias was potentially introduced. In addition, one study only reported univariate hazard ratio, which could introduce a bias toward overestimation of the prognostic role of SII. Finally, lymphocyte, neutrophil and platelet counts are commonly affected by the existence of infection, chemotherapy and other related factors and studies included in this metaanalysis did not strictly control these confounding factors. However, most cases included in the studies are patients who are going to have surgery and chemotherapy, which indicates that most cases were under non-infectious state when the peripheral blood for calculating SII was gathered. But we should admit that there are so many other factors such as the existence of dehydration, which is common but hard to control that affect the SII result.

In conclusion, our meta-analysis suggests elevated SII indicated poor prognosis and SII may serve as a cost-effective prognostic biomarker. Subgroup analyses by cancer sites identified consistent results in various

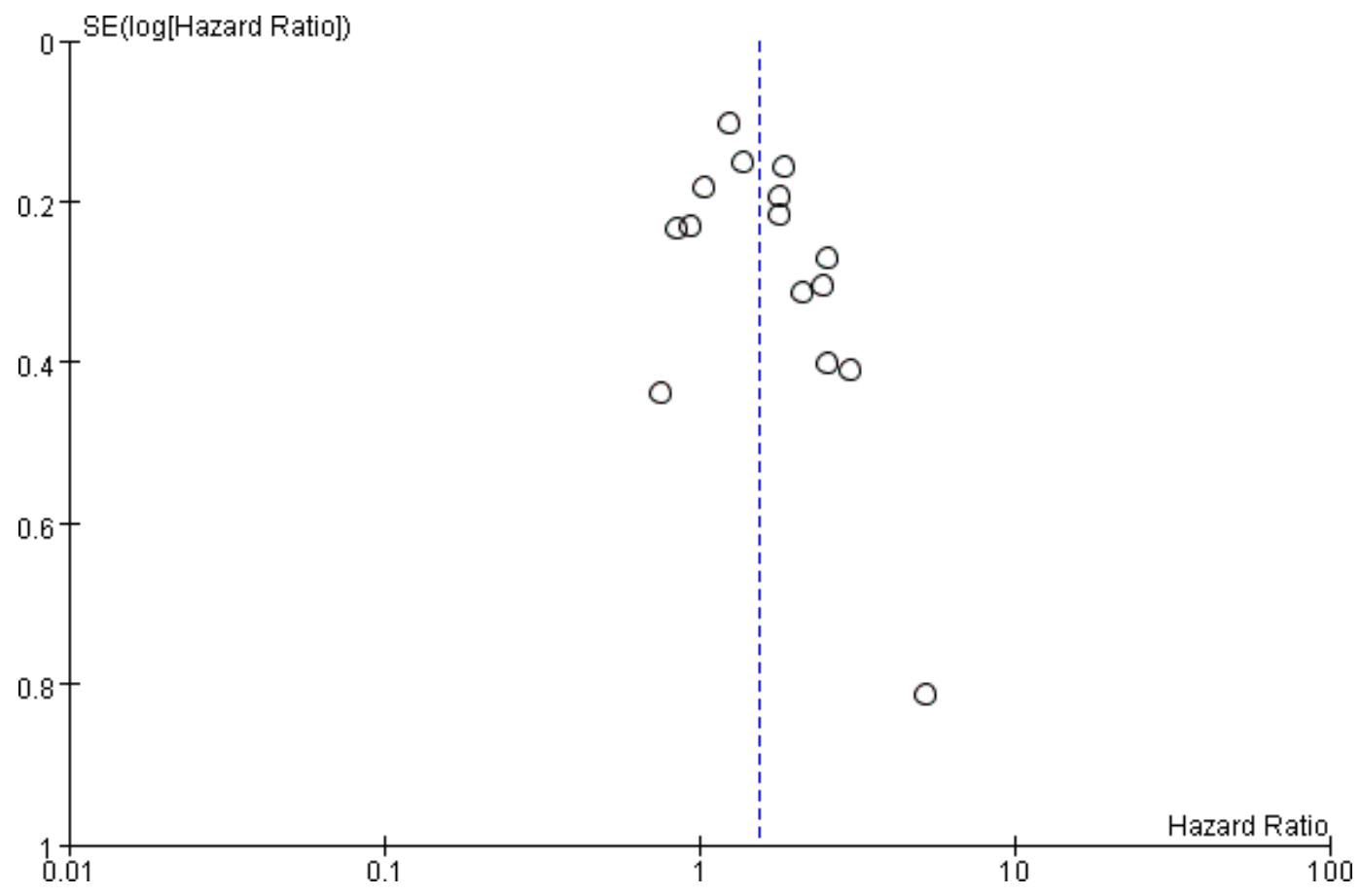

Figure 4: Funnel plots of hazard ratio for overall survival for high SII (horizontal axis) and the standard error (SE) for the hazard ratio (vertical axis). Each study is represented by one circle. The vertical line represents the pooled effect estimate. 
solid tumors, including hepatocellular carcinoma, gastrointestinal tract cancers, urinary cancers, small cell lung cancer and acral melanoma. The clinical significance of SII as a prognostic indicator must be further validated.

\section{CONFLICTS OF INTEREST}

The authors declare that there are no conflicts of interest regarding the publication of this paper.

\section{REFERENCES}

1. Grivennikov SI, Greten FR, Karin M. Immunity, inflammation, and cancer. Cell. 2010; 140:883-899.

2. Cools-Lartigue J, Spicer J, McDonald B, Gowing S, Chow S, Giannias B, Bourdeau F, Kubes P, Ferri L. Neutrophil extracellular traps sequester circulating tumor cells and promote metastasis. J Clin Invest. 2013 Jul 1. [Epub ahead of print].

3. Labelle M, Begum S, Hynes R. Direct Signaling between Platelets and Cancer Cells Induces an EpithelialMesenchymal-Like Transition and Promotes Metastasis. Cancer Cell. 2011; 20:576-590.

4. Mantovani A, Allavena P, Sica A, Balkwill F. Cancer-related inflammation. Nature. 2008; 454:436-444.

5. Proctor MJ, McMillan DC, Morrison DS, Fletcher CD, Horgan PG, Clarke SJ. A derived neutrophil to lymphocyte ratio predicts survival in patients with cancer. $\mathrm{Br} \mathrm{J}$ Cancer. 2012; 107:695-699.

6. Templeton AJ, Ace O, McNamara MG, Al-Mubarak M, VeraBadillo FE, Hermanns T, Seruga B, Ocana A, Tannock IF, Amir E. Prognostic role of platelet to lymphocyte ratio in solid tumors: a systematic review and meta-analysis. Cancer Epidemiol Biomarkers Prev. 2014; 23:1204-1212.

7. Templeton AJ, McNamara MG, Seruga B, Vera-Badillo FE, Aneja P, Ocana A, Leibowitz-Amit R, Sonpavde G, Knox JJ, Tran B, Tannock IF, Amir E. Prognostic role of neutrophilto-lymphocyte ratio in solid tumors: a systematic review and meta-analysis. J Natl Cancer Inst. 2014; 106:dju124.

8. Hu B, Yang XR, Xu Y, Sun YF, Sun C, Guo W, Zhang X, Wang WM, Qiu SJ, Zhou J, Fan J. Systemic immuneinflammation index predicts prognosis of patients after curative resection for hepatocellular carcinoma. Clin Cancer Res. 2014; 20:6212-6222.

9. Geng Y, Shao Y, Zhu D, Zheng X, Zhou Q, Zhou W, Ni X, Wu C, Jiang J. Systemic Immune-Inflammation Index Predicts Prognosis of Patients with Esophageal Squamous Cell Carcinoma: A Propensity Score-matched Analysis. Sci Rep. 2016; 6:39482.

10. Higgins JP, Thompson SG, Deeks JJ, Altman DG. Measuring inconsistency in meta-analyses. BMJ. 2003; 327:557-560.

11. Mantel N, Haenszel W. Statistical aspects of the analysis of data from retrospective studies of disease. J Natl Cancer Inst. 1959; 22:719-748.
12. DerSimonian R, Laird N. Meta-analysis in clinical trials. Control Clin Trials. 1986; 7:177-188.

13. Deeks JJ, Higgins JPT, Altman DG. Analysing Data and Undertaking Meta-Analyses. Cochrane Handbook for Systematic Reviews of Interventions: John Wiley \& Sons, Ltd). 2008; 243-296.

14. Dickersin K. The existence of publication bias and risk factors for its occurrence. JAMA. 1990; 263:1385-1389.

15. Egger M, Davey Smith G, Schneider M, Minder C. Bias in meta-analysis detected by a simple, graphical test. BMJ. 1997; 315:629-634.

16. Casadei Gardini A, Scarpi E, Faloppi L, Scartozzi M, Silvestris N, Santini D, de Stefano G, Marisi G, Negri FV, Foschi FG, Valgiusti M, Ercolani G, Frassineti GL. Immune inflammation indicators and implication for immune modulation strategies in advanced hepatocellular carcinoma patients receiving sorafenib. Oncotarget. 2016; 7:67142-67149. https://doi.org/10.18632/oncotarget.11565.

17. Feng JF, Chen S, Yang X. Systemic immune-inflammation index (SII) is a useful prognostic indicator for patients with squamous cell carcinoma of the esophagus. Medicine (Baltimore). 2017; 96:e5886.

18. Gao XH, Tian L, Wu J, Ma XL, Zhang CY, Zhou Y, Sun YF, Hu B, Qiu SJ, Zhou J, Fan J, Guo W, Yang XR. Circulating CD14+ HLA-DR-/low myeloid-derived suppressor cells predicted early recurrence of hepatocellular carcinoma after surgery. Hepatol Res. 2016; 47:1061-1071.

19. Ha H, Nam AR, Bang JH, Park JE, Kim TY, Lee KH, Han SW, Im SA, Kim TY, Bang YJ, Oh DY. Soluble programmed death-ligand 1 (sPDL1) and neutrophil-tolymphocyte ratio (NLR) predicts survival in advanced biliary tract cancer patients treated with palliative chemotherapy. Oncotarget. 2016; 7:76604-76612. https:// doi.org/10.18632/oncotarget.12810.

20. Hong X, Cui B, Wang M, Yang Z, Wang L, Xu Q. Systemic Immune-inflammation Index, Based on Platelet Counts and Neutrophil-Lymphocyte Ratio, Is Useful for Predicting Prognosis in Small Cell Lung Cancer. Tohoku J Exp Med. 2015; 236:297-304.

21. Huang L, Liu S, Lei Y, Wang K, Xu M, Chen Y, Liu B, Chen Y, Fu Q, Zhang P, Qin K, Cai Y, Fu S, et al. Systemic immune-inflammation index, thymidine phosphorylase and survival of localized gastric cancer patients after curative resection. Oncotarget. 2016; 7:44185-44193. https://doi. org/10.18632/oncotarget.9923.

22. Jin H, Pang Q, Liu H, Li Z, Wang Y, Lu Y, Zhou L, Pan H, Huang W. Prognostic value of inflammation-based markers in patients with recurrent malignant obstructive jaundice treated by reimplantation of biliary metal stents: A retrospective observational study. Medicine (Baltimore). 2017; 96:e5895.

23. Liu X, Sun X, Liu J, Kong P, Chen S, Zhan Y, Xu D. Preoperative C-Reactive Protein/Albumin Ratio Predicts Prognosis of Patients after Curative Resection for Gastric Cancer. Transl Oncol. 2015; 8:339-345. 
24. Lolli C, Basso U, Derosa L, Scarpi E, Sava T, Santoni M, Crabb SJ, Massari F, Aieta M, Conteduca V, Maruzzo M, La Russa F, Wheater M, et al. Systemic immuneinflammation index predicts the clinical outcome in patients with metastatic renal cell cancer treated with sunitinib. Oncotarget. 2016; 7:54564-54571. https://doi. org/10.18632/oncotarget.10515.

25. Lolli C, Caffo O, Scarpi E, Aieta M, Conteduca V, Maines F, Bianchi E, Massari F, Veccia A, Chiuri VE, Facchini G, De Giorgi U. Systemic Immune-Inflammation Index Predicts the Clinical Outcome in Patients with mCRPC Treated with Abiraterone. Front Pharmacol. 2016; 7:376.

26. Passardi A, Scarpi E, Cavanna L, Dall'Agata M, Tassinari D, Leo S, Bernardini I, Gelsomino F, Tamberi S, Brandes AA, Tenti E, Vespignani R, Frassineti GL, et al. Inflammatory indexes as predictors of prognosis and bevacizumab efficacy in patients with metastatic colorectal cancer. Oncotarget. 2016; 7:33210-33219. https://doi.org/10.18632/ oncotarget.8901.

27. Yang Z, Zhang J, Lu Y, Xu Q, Tang B, Wang Q, Zhang W, Chen S, Lu L, Chen X. Aspartate aminotransferaselymphocyte ratio index and systemic immuneinflammation index predict overall survival in HBV-related hepatocellular carcinoma patients after transcatheter arterial chemoembolization. Oncotarget. 2015; 6:43090-43098. https://doi.org/10.18632/oncotarget.5719.

28. Yu J, Li SM, Kong Y, Si L, Sheng X, Chi Z, Cui C, Guo J. Association of immune-inflammation index with outcome of high-risk acral melanoma patients treated with adjuvant highdose interferon. Journal of Clinical Oncology. 2016; 34.

29. Aggarwal BB, Vijayalekshmi RV, Sung B. Targeting inflammatory pathways for prevention and therapy of cancer: short-term friend, long-term foe. Clin Cancer Res. $2009 ; 15: 425-430$.
30. Levine MN, Browman GP, Gent M, Roberts R, Goodyear M. When is a prognostic factor useful? A guide for the perplexed. J Clin Oncol. 1991; 9:348-356.

31. Chen HC, Lin HC, Liu CY, Wang CH, Hwang T, Huang TT, Lin CH, Kuo HP. Neutrophil elastase induces IL-8 synthesis by lung epithelial cells via the mitogen-activated protein kinase pathway. Journal of Biomedical Science. 2004; 11:49-58.

32. De Larco JE, Wuertz BR, Furcht LT. The potential role of neutrophils in promoting the metastatic phenotype of tumors releasing interleukin-8. Clinical Cancer Research. 2004; 10:4895-4900.

33. Houghton AM, Rzymkiewicz DM, Ji H, Gregory AD, Egea EE, Metz HE, Stolz DB, Land SR, Marconcini LA, Kliment CR, Jenkins KM, Beaulieu KA, Mouded M, et al. Neutrophil elastase-mediated degradation of IRS-1 accelerates lung tumor growth. Nature Medicine. 2010; 16:219-223.

34. Teramukai S, Kitano T, Kishida Y, Kawahara M, Kubota K, Komuta K, Minato K, Mio T, Fujita Y, Yonei T, Nakano K, Tsuboi M, Shibata K, et al. Pretreatment neutrophil count as an independent prognostic factor in advanced non-smallcell lung cancer: an analysis of Japan Multinational Trial Organisation LC00-03. Eur J Cancer. 2009; 45:1950-1958.

35. Labelle M, Begum S, Hynes RO. Direct signaling between platelets and cancer cells induces an epithelialmesenchymal-like transition and promotes metastasis. Cancer Cell. 2011; 20:576-590. 